\title{
Extended Hopfield Models for Combinatorial Optimization
}

\author{
Armelle Le Gall and Vassilis Zissimopoulos
}

\begin{abstract}
The extended Hopfield neural network proposed by Abe et al. for solving combinatorial optimization problems with equality and/or inequality constraints has the drawback of being frequently stabilized in states with neurons of ambiguous classification as active or inactive. We introduce in the model a competitive activation mechanism and we derive a new expression of the penalty energy allowing us to reduce significantly the number of neurons with intermediate level of activations. The new version of the model is validated experimentally on the set covering problem. Our results confirm the importance of instituting competitive activation mechanisms in Hopfield neuralnetwork models.
\end{abstract}

Index Terms-Activation mechanism, combinatorial optimization, competitive heuristic, Hopfield neural network, inequality constraints, set covering problem.

\section{INTRODUCTION}

A BE et al. [2] have given an extension of the Hopfield model [1], [5] in order to handle equality or/and inequality constraints. The extended Hopfield model (EHM) introduces in the objective function some additional energy terms which penalize any infeasible state. For determining an appropriate expression for the penalty energy in the inequality constraints case, each inequality constraint is converted to an equality constraint. This is obtained by introducing an additional variable which is managed by a new neuron. Each new neuron is connected to the initial neurons where their corresponding variables occur in its linear combination. The disadvantage of the EHM is to produce in many cases uninterpretable stable solutions, that is, some neurons with activation levels far from the suitable ones, i.e., zero and one. The aim of this paper is to reduce such undesirable situations and to improve EHM performance by introducing a competitive activation mechanism in the model. In a relative work, to handle inequality constraints, Ohlsonn et al. [9] have used another expression for the additional energy term and in [10] and [11] a Potts glass theory technique using mean field theory has been developed and tested on the knapsack problem and the assignment problem.

Here, we use an analogy between handling inequality constraints strategy and an allocation problem of limited resources. For each constraint a fictional resource, the amount of which represents the degree of constraint unsatisfaction, is attributed

Manuscript received December 17, 1996; revised January 4, 1998.

A. LeGall is with the LRI, CNRS-URA 410, Bât 490, Université de Paris Sud, 91405 Orsay Cedex, France.

V. Zissimopoulos is with the LIPN, CNRS-URA 1507, Université de Paris Nord, 93430 Villetaneuse, France.

Publisher Item Identifier S 1045-9227(99)00939-X. to the corresponding new neuron. At its present form, the EHM takes as resource amounts the differences between the bounds and the current values of the linear combinations. Then, it distributes them proportionably to the receiving neuron activations.

In order to reinforce in EHM neurons repartition into two categories: active or inactive, we institute an inequitable way to distribute the resources. Henceforth, in the lowerbounded constraint case (positive resources) the most active neurons are being in favor whereas in the upper-bounded constraint case (negative resources) there are the most inactive neurons that are favorized. These inequitable allocation rules create inhibitory relationships between neurons which are in competition to acquire the same limited resource. For the case of positive resources Reggia has defined [12] the rules which must govern resources allocation in order to select a limited number of neurons as competition winners. This allocation method, reported as competitive activation mechanism, is well suited to resolve unexclusive in nature competitions, that is, competitions eventually requiring some collaborations between competitors for elaborating a global solution. The competitions implemented to satisfy inequality constraints are owned to this category.

Here, we extend this competitive activation mechanism for dealing with negative resources and we deduce a new expression for the energy term which integrates the inequality constraints in the Hopfield model. This energy generates activation rules ensuring a better neurons repartition to two sets. The set including neurons with near to zero activation and the set including neurons with near to one activation. We show also how to evaluate the integration weights of the new penalty energy essentially based on the idea developed by Abe [1] for dealing with the equality constraints on the traveling salesman problem. We obtain in this way for a combinatorial optimization problem that any infeasible solution located on a vertex in the proximity of a feasible solution can not be a local minimum of the network's energy.

The new method for handling inequality constraints is validated experimentally on the set covering problem (SCP) [6]. In the next section we describe briefly the EHM. In Section III we discuss different competitive mechanisms and present an extension of the EHM including competitive activation mechanisms. We derive the new activation rules and we show how to define the main involved parameters. In Section IV we discuss the set covering problem and we adapt the neural model for solving it. In Section V we present an extensive experimental study which confirms the utility of the instituted competitive activation mechanism. 


\section{The ExTEnded Hopfield Model}

The original Hopfield model is a neural-network model adequate for solving optimization problems which can be expressed by a quadratic function of the form

$$
\begin{aligned}
& \text { minimize } E_{1}(x)=\frac{1}{2} x^{t} P x+q^{t} x \\
& \text { with } x \in\{0,1\}^{n}
\end{aligned}
$$

where $x=\left(x_{1}, \cdots, x_{n}\right)^{t}$ is the vector of problem variables, $q$ a $n$th constant vector and $P$ a symmetric $n \times n$ matrix with $P_{i i}=0, i=1, \cdots, n[5]$.

The EHM proposed by Abe et al. [2] is able to handle the following constraints.

- The $l$ equality constraints: $r_{i}^{t} x=s_{i}, i=1, \cdots, l$, where $r_{i}^{t}=\left(r_{1 i}, \cdots, r_{n i}\right)$.

- The $k$ inequality constraints: $w_{i}^{t} x \leq d_{i}$ or $w_{i}^{t} x \geq d_{i}$ where $d_{i}>0$ and $w_{i}^{t}=\left(w_{1 i}, \cdots, w_{n i}\right), w_{j i} \in \mathcal{R}, j=$ $1, \cdots, n, i=1, \cdots, k$.

We describe the EHM with a slight modification allowing us to handle also inequality constraints with $d_{i}=0$. This case will be useful later (Sections IV and V) and it affects the definition domain of the introduced new variables.

Handling constraints implies the weighted insertion of two energy terms into the initial objective function $E_{1}$. The first term, denoted by $E_{2}$, is related to the equality constraints and the second term, denoted by $E_{3}$, is related to the inequality constraints. The minimizing by the network function is given by $E=A E_{1}+B E_{2}+C E_{3}$ where $A, B$, and $C$ are strictly positive weights.

Let us first consider the equality constraints. We are looking for a function $E_{2}$ of $x$ whom the minimum is obtained only when every equality constraint $r_{i}^{t} x=s_{i}, i=1, \cdots, l$, is satisfied. It is defined as follows:

$$
E_{2}=\sum_{i=1}^{l}\left[\frac{1}{2}\left(r_{i}^{t} x\right)^{2}-s_{i} r_{i}^{t} x\right] .
$$

By denoting $R=\sum_{i=1}^{l} r_{i} r_{i}^{t}$ and $s=-\sum_{i=1}^{l} s_{i} r_{i}, E_{2}$ can been written in a matricial form: $E_{2}=1 / 2 x^{t} R x+s^{t} x$. Let $T^{\prime}=A P+B R$ and $b=A q+B s$. Then, the function $A E_{1}+B E_{2}=1 / 2 x^{t} T^{\prime} x+b^{t} x$ is a quadratic function and so, the initial Hopfield model can be used for handling equality constraints.

For handling the $k$ inequality constraints we convert them into equality constraints by introducing new variables $y_{i}$, for $i=1, \cdots, k$, brought together into the vector $y=$ $\left(y_{1}, \cdots, y_{k}\right)^{t}$. Then, it is easy to prove the following lemma.

Lemma 1: For $i$ varying from one to $k$, the inequality constraint $w_{i}^{t} x \leq d_{i}$ or $w_{i}^{t} x \geq d_{i}$ with nonnegative bound $d_{i}$ is equivalent to the equality constraint $d_{i}^{\prime} y_{i}-w_{i}^{t} x=0$ with

and

$$
d_{i}^{\prime}= \begin{cases}d_{i}, & \text { if } d_{i} \neq 0 \\ 1, & \text { otherwise }\end{cases}
$$

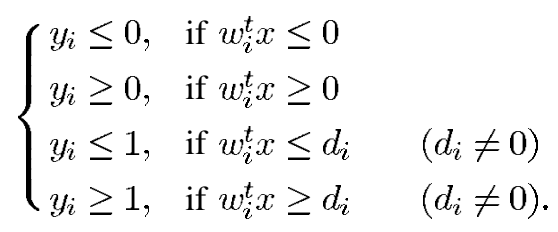

This preliminary step allows us to use the same schema as the one used for the equality constraints. So, the energy function $E_{3}$ is given by

$$
E_{3}(x, y)=\frac{1}{2} \sum_{i=1}^{k}\left(w_{i}^{t} x-d_{i}^{\prime} y_{i}\right)^{2} .
$$

With the notations

$$
\begin{aligned}
V^{\prime} & =-\left(d_{1}^{\prime} w_{1}, \cdots, d_{k}^{\prime} w_{k}\right): n \times k \text { matrix } \\
W^{\prime} & =\sum_{i=1}^{k} w_{i} w_{i}^{t}: n \times n \text { matrix } \\
D^{\prime} & =\left(\begin{array}{ccc}
d_{1}^{2} & \cdots & 0 \\
0 & & d_{k}^{\prime 2}
\end{array}\right): k \times k \text { diagonal matrix. }
\end{aligned}
$$

$E_{3}$ can be written as follows:

$$
E_{3}(x, y)=\frac{1}{2}\left(x^{t}, y^{t}\right)\left(\begin{array}{ll}
W^{\prime} & V^{\prime} \\
V^{\prime} & D^{\prime}
\end{array}\right)\left(\begin{array}{l}
x \\
y
\end{array}\right) .
$$

By putting $W=C W^{\prime}, V=C V^{\prime}, D=C D^{\prime}$ and $T=$ $T^{\prime}+W$, the global energy $E=A E_{1}+B E_{2}+C E_{3}$ that the model must to minimize is written

$$
E(x, y)=\frac{1}{2}\left(x^{t}, y^{t}\right)\left(\begin{array}{cc}
T & V \\
V^{t} & D
\end{array}\right)\left(\begin{array}{l}
x \\
y
\end{array}\right)+b^{t} x .
$$

We obtain for the EHM the following result handling also inequality constraints with $d_{i}=0$.

Proposition 2: A two layers connectionist model whom the behavior is controlled by the dynamic system

$$
\begin{aligned}
\left(\begin{array}{l}
d u / d t \\
d v / d t
\end{array}\right) & =-\left(\begin{array}{l}
\partial E / \partial x \\
\partial E / \partial y
\end{array}\right) \\
& =-\left(\begin{array}{cc}
T & V \\
V & D
\end{array}\right)\left(\begin{array}{l}
x \\
y
\end{array}\right)-\left(\begin{array}{l}
b \\
0
\end{array}\right)
\end{aligned}
$$

with $u \in(-\infty,+\infty)^{n}$ and $x \in(0,1)^{n}$ the vectors of internal and coming-out variables for the first layer

$$
\forall j=1, \cdots, n x_{j}=\frac{1}{2}\left(1+\tanh \frac{u_{j}}{\tau_{j}}\right) \quad\left(\text { with } \tau_{j}>0\right)
$$

and $v \in(-\infty,+\infty)^{k}$ and $y$ the vectors of internal and coming-out variables for the second layer

$$
\begin{aligned}
\forall i= & 1, \cdots, k \\
y_{i} & =\left\{\begin{array}{ll}
-\exp \left(-v_{i} / \rho_{i}\right), & \text { if } y_{i} \leq 0 \\
\exp \left(v_{i} / \rho_{i}\right), & \text { if } y_{i} \geq 0 \\
1-\exp \left(-v_{i} / \rho_{i}\right), & \text { if } y_{i} \leq 1 \\
1+\exp \left(v_{i} / \rho_{i}\right), & \text { if } y_{i} \geq 1
\end{array} \quad\right. \text { (with }
\end{aligned}
$$

converges to a local minimum of the function $E$.

The convergence proof consists to establish that the function $E$ is a Lyapunov function for the dynamic system defined by (5). In [1] and [2], the conditions for the system to converge to a vertex, a point on the surface or an interior point in the $n$-dimensional hypercube have been studied by an eigenvalue analysis of the linearized system.

In summary, the EHM has two layers. The first layer includes $n$ neurons $\Xi_{j}, j=1, \cdots, n$, interconnected following the matrix $T$. Each such neuron is associated to a variable $x_{j}$. The second layer includes $k$ neurons $\Upsilon_{i}, i=1, \cdots, k$, 
each one corresponding to the new variable $y_{i}$. A neuron $\Upsilon_{i}$ is linked to the $\Xi_{j}$ neuron with a synaptic weight $w_{j i}$ which is exactly the coefficient of the variable $x_{j}$ in the linear combination $w_{i}^{t} x$. The model operates in a synchronous mode alternatively on the two layers until reaching a stable state. The rules which govern the evolution of neurons $\Xi_{j}$ and $\Upsilon_{i}$ are, respectively,

$$
\begin{aligned}
\frac{d x_{j}}{d t}= & \frac{2}{\tau_{j}}\left(C \sum_{i=1, \cdots, k} w_{j i} x_{j}\left[d_{i}^{\prime} y_{i}-w_{i}^{t} x\right]-\left[T_{j}^{\prime} x+b_{j}\right] x_{j}\right) \\
& \cdot\left(1-x_{j}\right) \\
\frac{d y_{i}}{d t}= & -C d_{i}^{\prime} f\left(y_{i}\right)\left(d_{i}^{\prime} y_{i}-w_{i}^{t} x\right)
\end{aligned}
$$

with

$$
f\left(y_{i}\right)= \begin{cases}-y_{i} / \rho_{i} & \text { if } y_{i} \leq 0 \\ y_{i} / \rho_{i} & \text { if } y_{i} \geq 0 \\ \left(1-y_{i}\right) / \rho_{i} & \text { if } y_{i} \leq 1 \\ \left(y_{i}-1\right) / \rho_{i} & \text { if } y_{i} \geq 1\end{cases}
$$

\section{Competitive ACTIVATION MECHANISMS}

In order to estimate the capacity of the EHM to share out the neurons in active and in inactive ones, we consider an analogy between the inequality constraints and an allocation problem of limited resources $R_{i}=d_{i}^{\prime} y_{i}-w_{i}^{t} x$ for $i=1, \cdots, k$. Each neuron $\Upsilon_{i}$ evaluates the amount of its resource which is interpreted as a constraint unsatisfaction measure. An analysis of the EHM dynamic shows that, for a satisfied constraint, the unsatisfaction degree tends to zero because $d_{i}^{\prime} y_{i}$ converges asymptotically to $w_{i}^{t} x$. For an unsatisfied constraint, $d_{i}^{\prime} y_{i}$ converges asymptotically to $d_{i}$, and so, $R_{i}$ converges to $d_{i}-$ $w_{i}^{t} x$. By distributing its resource to the neighbors $\Xi_{j}$, a neuron $\Upsilon_{i}$ influences the evolution of the network toward to a state which should satisfy its corresponding constraint. Resources distribution is realized by sending out flows, out $\Upsilon_{i} \rightarrow \Xi_{j}$, which are proportional to the amount $R_{i}$ and to the activation level $x_{j}$ of the receiving neuron

$$
\forall i=1, \cdots, k, \forall j=1, \cdots, n, \text { out }_{\Upsilon_{i} \rightarrow \Xi_{j}}=w_{j i} x_{j} R_{i} .
$$

Notice that a such distribution does not produce the desirable neurons sharing out. Indeed, when $w_{i}^{t} x \geq d_{i}$ (positive resource) the advantage offered to a strongly activated receiving neuron $\Xi_{j}$ is almost eliminated by the inhibition term $\left[T_{j}^{\prime} x+b_{j}\right] x_{j}$ which is also proportional to its self activation level $x_{j}$. Therefore, the activations of the less active neurons progress as far as the ones of the more active neurons. So, many neurons after networks stability will have activation values which are far from both one and zero. Also, when $w_{i}^{t} x \leq d_{i}$ (negative resource) the neurons which must receive large negative flows are the neurons with weak activations in order to ensure their activation decreament and let the neurons with strong activations near the bound one. However, the model in its present form tends to decrease the activation of the strongly activated neurons by sending to them large negative flows. As a consequence, a stable state will still include neurons with uninterpretable activations.
An inequitable allocation of resources achievable by instituting competitions between all $\Xi_{j}$ neurons owning a common resource aims to remedy this drawback.

Definition 1: Two neurons are said to be competitors if the gain of one occurs at the expense of the other, i.e., their functional relationships are inhibitory in nature [4].

This definition of competition in neural networks, due to Grossberg, indicates inhibitory interactions but it says nothing about the underlying mechanisms involved in producing those inhibitory interactions. The direct or antagonist competition is a well-known competition mechanism. It occurs when an active entity directly interacts with rival entities by sending out negative flow in order to suppress theirs activities. Since, all neurons having lost a competition can not become active. This mechanism generates a single winner-takes-all behavior. But, in some applications, in order to produce a global solution some competitors must cooperate. For capturing competitive but cooperative relationships between neighbors and producing so a multiple-winners-take-all behavior, the following mechanism, called indirect competition or competitive activation mechanism (cam) [8], [12], [13], could be more adapted. It occurs when two rivals require and consume the same limited resource. Under the hypothesis that the connection weights and the resources are not negative, for sharing out the competitors between the losers and the winners, the resources must respect the following principles.

- The activation flows should be representative of the way by which the emitting neuron wishes allocating its resource between its neighboring neurons. For this, the activation flows are positive and their amount is equal to the resource.

- An inhibition term in the activation rule should decrease the activations of the neurons not acquiring enough resources. This term prevents network's saturation. For a neuron $\Xi_{j}$, the activation rule looks as: $d x_{j} / d t=$ $\left[I n_{j}-I h_{j} x_{j}\right]\left(1-x_{j}\right)$ with $I n_{j}$ the sum of input activation flows and $I h_{j} x_{j}$ its inhibition term. The factor $\left(1-x_{j}\right)$ and the inhibition term, which is proportional to its self activation $x_{j}$, are used to prevent the activations from overstepping their upper or lower bound.

- Large activation flows should be sent to the stronger activated neurons in order to they acquire the active level in spite of their inhibition terms increament.

We return now in the combinatorial optimization problem and the EHM. In general, many variables equal to one are needed to satisfy a lower-bounded inequality constraint. By using the EHM this type of constraint is solved by generating positive resources. So, the competitive mechanism is well suited to use. But, for upper-bounded inequality constraints the resources are negative and their satisfaction, in general, needs several variables equal to zero. So, it is worthful to extend the cam for handling also negative resources. This can be done by sending inhibitory flows tending to decrease the activation of the receiving neurons preferably those having weak activations. In summary, ensuring cam in the EHM the following conditions are required. These conditions reflect the previously described principles of cam but with positive and 
negative resources.

1)

$$
\forall \Xi_{j}, \text { out }_{\Upsilon_{i} \rightarrow \Xi_{j}} \begin{cases}\geq 0, & \text { if the constraint type } \\ & \text { is } w_{i}^{t} x \geq d_{i} \quad\left(R_{i} \geq 0\right) \\ \leq 0, & \text { if the constraint type } \\ & \text { is } w_{i}^{t} x \leq d_{i} \quad\left(R_{i} \leq 0\right) .\end{cases}
$$

2) out $_{\Upsilon_{i}}=\sum_{j=1}^{n}$ out $_{\Upsilon_{i} \rightarrow \Xi_{j}}=R_{i}$.

3) If $w_{j i}=w_{p i} \neq 0$ and $x_{j}>x_{p}$ then

$$
\frac{\operatorname{out}_{\Upsilon_{i} \rightarrow \Xi_{j}}}{\operatorname{out}_{\left(\Upsilon_{i} \rightarrow \Xi_{p}\right.}} \begin{cases}>x_{j} / x_{p}, & \text { if } R_{i}>0 \\ <x_{j} / x_{p}, & \text { if } R_{i}<0 .\end{cases}
$$

It can be seen that in the EHM the flows out $\Upsilon_{i} \rightarrow \Xi_{j}=w_{j i} x_{j} R_{i}$ which distribute the resource $R_{i}=d_{i}^{\prime} y_{i}-w_{i}^{t} x$ do not respect the second and the third properties of cam. In fact, the sign of the sent flows is identical to the one of the resource $R_{i}$ but the global amount emitting by the neuron $\Upsilon_{i}$, i.e., out $\Upsilon_{i}=w_{i}^{t} x R_{i}$, is not equal to the resource value. For the third condition, let consider two competitors $\Xi_{j}$ and $\Xi_{p}$ requiring the same resource $R_{i}$ and having $w_{j i}=w_{p i}$. Clearly, they receive flows with a ratio equal to the ratio of their activation levels

$$
\forall \Xi_{j}, \Xi_{p}, w_{j i}=w_{p i}, \frac{\text { out }_{\Upsilon_{i} \rightarrow \Xi_{j}}}{\operatorname{out}_{\Upsilon_{i} \rightarrow \Xi_{p}}}=\frac{x_{j}}{x_{p}} .
$$

Under only the influence of the neuron $\Upsilon_{i}$, if the two activation levels $x_{j}$ and $x_{p}$ are almost identical, the two neurons are going to evolute identically, since we have $d x_{j} / d t \simeq d x_{p} / d t$. In the next section, we show how to ensure in EHM the two not respected conditions of cam.

\section{A. The Competitive Activation Extended Hopfield Model}

The second condition, requiring that neuron $\Upsilon_{i}$ sends out a global quantity of flows equal to its resource amount $R_{i}$, is simply obtained by normalization of the flows out $\Upsilon_{i} \rightarrow \Xi_{j}$, i.e., by sending flows proportional to the rate $w_{j i} x_{j} / w_{i}^{t} x, i=$ $1, \cdots, k, j=1, \cdots, n$. However, a such expression is partially correct; it still remains to favor the best competitors, that is, the most active (respectively, the less active) in the lower bounded inequality constraints case (respectively, the upper bounded inequality constraints). For this, before the normalization phase, we raise into the linear combination $w_{i}^{t} x$ the variables $x_{j}$ to a power $\alpha_{i}$. This modification generates flows proportional to $w_{j i} x_{j}^{\alpha_{i}} / w_{i}^{t} x^{\alpha_{i}}$ where $x^{\alpha_{i}}$ denotes the vector $\left(x_{1}^{\alpha_{i}}, \cdots, x_{n}^{\alpha_{i}}\right)^{t}$. The power $\alpha_{i}$ is allowing us to accentuate the existing gap between the activation levels of neurons $\Xi_{j}$. Indeed

$$
\begin{aligned}
\text { if } w_{j i} & =w_{p i} \neq 0 \text { and } x_{j}>x_{p} \text { then } \\
\frac{\text { out }_{\Upsilon_{i} \rightarrow \Xi_{j}}}{{\text { out } \Upsilon_{i} \rightarrow \Xi_{p}}} & =\left[\frac{x_{j}}{x_{p}}\right]^{\alpha_{i}} \begin{cases}>x_{j} / x_{p}, & \text { if } \alpha_{i}>1 \\
<x_{j} / x_{p}, & \text { if } 0<\alpha_{i}<1 .\end{cases}
\end{aligned}
$$

In this expression, if the type of $i$ th constraint is $w_{i}^{t} x \geq d_{i}$ $\left(w_{i}^{t} x \leq d_{i}\right)$ the condition $\alpha_{i}>1$ (respectively, $0<\alpha_{i}<1$ ) ensures the third condition of cam.

Notice that the contribution of the variable $x_{j}$ is always bounded by the weight $w_{j i}$. So, for the lower bounded constraints $\left(w_{i}^{t} x \geq d_{i}\right)$ the linear combination is lower estimated, i.e., $w_{i}^{t} x^{\alpha_{i}}<w_{i}^{t} x$ since $\alpha_{i}>1$. For the upper bounded constraints $\left(w_{i}^{t} x \leq d_{i}\right)$ the linear combination is upper estimated, i.e., $w_{i}^{t} x^{\alpha_{i}}>w_{i}^{t} x$ since $0<\alpha_{i}<1$. Thereby, the inequality constraints satisfaction requires a large number of variables to tend to an activation level sufficiently near to one or zero. Intuitively, these observations show that the instituting cam contributes significantly to direct network's convergence to a vertex of the hypercube.

Let now study the dynamics of the new extended Hopfield model (EHM) cam with the instituted cam. The normalization of the activation flows is obtained by applying the logarithm function on each term of the inequality constraints before their transformations into equality constraints.

Lemma 3: The $k$ inequality constraints $w_{i}^{t} x \leq d_{i}$ or $w_{i}^{t} x \geq$ $d_{i}$ with bounds $d_{i} \neq 0$ are equivalent to the equality constraints $d_{i}^{\prime} y_{i}-\ln \left(w_{i}^{t} x^{\alpha_{i}}\right)=0$ for $i=1, \cdots, k$ provided that

$$
d_{i}^{\prime}= \begin{cases}1, & \text { if } d_{i}=1 \\ \ln \left(d_{i}\right), & \text { otherwise }\end{cases}
$$

and

$$
\begin{cases}y_{i} \leq 0, & \text { if } w_{i}^{t} x \leq 1 \\ y_{i} \geq 0, & \text { if } w_{i}^{t} x \geq 1 \\ y_{i} \leq 1, & \text { if } w_{i}^{t} x \leq d_{i} \quad\left(d_{i} \neq 1\right) \\ y_{i} \geq 1, & \text { if } w_{i}^{t} x \geq d_{i} \quad\left(d_{i} \neq 1\right) .\end{cases}
$$

The corresponding to the inequality constraints new energy in the (EHM) cam is now written

$$
\operatorname{Ecam}_{3}(x, y)=\frac{1}{2} \sum_{i=1}^{k} \frac{C_{i}}{\alpha_{i}}\left[\ln \left(w_{i}^{t} x^{\alpha_{i}}\right)-d_{i}^{\prime} y_{i}\right]^{2} .
$$

The Proposition 2 can be stated as follows.

Proposition 4: A two layers connectionist model whom the behavior is controlled by the dynamic system

$$
\begin{aligned}
& \left(\begin{array}{l}
d u / d t \\
d v / d t
\end{array}\right) \\
& =-\left(\begin{array}{l}
\partial E c a m / \partial x \\
\partial E c a m / \partial y
\end{array}\right) \\
& =-\left(\begin{array}{l}
{\left[T^{\prime} x+b\right]+\sum_{i=1}^{k} C_{i} \frac{w_{j i} x_{j}^{\alpha_{j}-1}}{w_{i}^{t} x_{\alpha_{i}}}\left[\ln \left(w_{i}^{t} x^{\alpha_{i}}\right)-d_{i}^{\prime} y_{i}\right]} \\
\cdot \frac{C_{i}}{\alpha_{i}} d_{i}^{\prime}\left[d_{i}^{\prime} y_{i}-\ln \left(w_{i}^{t} x_{\alpha_{i}}\right)\right]
\end{array}\right)
\end{aligned}
$$

with $u \in(-\infty,+\infty)^{n}$ and $x \in(0,1)^{n}$ the vectors of internal and coming-out variables for the first layer: $\forall j=$ $1, \cdots, n x_{j}=\frac{1}{2}\left[1+\tanh \left(u_{j} / \tau_{j}\right)\right]$ (with $\tau_{j}>0$ ) and $v \in(-\infty,+\infty)^{k}$ and $y$ the vectors of internal and coming-out variables for the second layer: $\forall i=1, \cdots, k$

$$
y_{i}=\left\{\begin{array}{lll}
-\exp \left(-v_{i} / \rho_{i}\right), & \text { if } y_{i} \leq 0 \\
\exp \left(v_{i} / \rho_{i}\right), & \text { if } y_{i} \geq 0 & \text { (with } \\
1-\exp \left(-v_{i} / \rho_{i}\right), & \text { if } y_{i} \leq 1 & \left.\rho_{i}>0\right) \\
1+\exp \left(v_{i} / \rho_{i}\right), & \text { if } y_{i} \geq 1 &
\end{array}\right.
$$

converges to a local minimum of the function

$$
\begin{aligned}
\operatorname{Ecam}(x, y)= & A E_{1}(x)+B E_{2}(x)+\operatorname{Ecam}_{3}(x, y) \\
= & \frac{1}{2} x^{t} T^{\prime} x+b^{t} x+\frac{1}{2} \sum_{i=1}^{k} \frac{C_{i}}{\alpha_{i}} \\
& \cdot\left[\ln \left(w_{i}^{t} x^{\alpha_{i}}\right)-d_{i}^{\prime} y_{i}\right]^{2} .
\end{aligned}
$$


The convergence proof is similar to this one in [1] and [2] and the activation rules for the neurons $\Xi_{j}$ and $\Upsilon_{i}$ have as follows:

$$
\begin{gathered}
\frac{d x_{j}}{d t}=\frac{2}{\tau_{j}}\left(\sum_{i=1, \cdots, k} C_{i} \frac{w_{j i} x_{j}^{\alpha_{i}}}{w_{i}^{t} x^{\alpha_{i}}}\left[d_{i}^{\prime} y_{i}-\ln \left(w_{i}^{t} x^{\alpha_{i}}\right)\right]\right. \\
\left.-\left[T_{j}^{\prime} x+b_{j}\right] x_{j}\right)\left(1-x_{j}\right) \\
\frac{d y_{i}}{d t}=-\frac{C_{i}}{\alpha_{i}} d_{i}^{\prime} f\left(y_{i}\right)\left(d_{i}^{\prime} y_{i}-\ln \left(w_{i}^{t} x^{\alpha_{i}}\right)\right) .
\end{gathered}
$$

Then, the new expressions of the resources $R_{i}^{\alpha_{i}}$ attributed to the neurons $\Upsilon_{i}$ and the activation flows out $\Upsilon_{i} \rightarrow \Xi_{j}$ spread from $\Upsilon_{i}$ to neurons $\Xi_{j}$ are equal to

$$
\begin{aligned}
R_{i}^{\alpha_{i}} & =d_{i}^{\prime} y_{i}-\ln \left(w_{i}^{t} x^{\alpha_{i}}\right), \\
\text { out }_{\Upsilon_{i} \rightarrow \Xi_{j}} & =\frac{w_{j i} x_{j}^{\alpha_{i}}}{w_{i}^{t} x^{\alpha_{i}}}\left[d_{i}^{\prime} y_{i}-\ln \left(w_{i}^{t} x^{\alpha_{i}}\right)\right] \\
& =\frac{w_{j i} x_{j}^{\alpha_{i}}}{w_{i}^{t} x^{\alpha_{i}}} R_{i}^{\alpha_{i}} .
\end{aligned}
$$

By checking the three conditions it can easily be seen that the (EHM) cam incorporates the cam.

\section{B. Weights Determination}

Following the idea of Abè [1] for defining the value of the weight $B$ in relation to $A$ for the equality constraints on the traveling salesman problem, we prove the following result for the weights $A, B$, and $C_{i}, i=1, \cdots, k$, which is valid for a combinatorial optimization problem with equality and inequality constraints. Let us consider two adjacent vertices $c$ and $c(j)$ in the hypercube $[0,1]^{n}$ which differ only on their $j$ th component.

Theorem 5: If the coefficients $w_{j i}$ and $d_{i}$ are nonnegative integers and the weights $A, B$, and $C_{i}$ are defined with respect to

$$
\begin{aligned}
& B>2 A \frac{\max _{c \in \Gamma} \max _{j=1, \cdots, n}\left(0, E_{1}(c)-E_{1}(c(j))\right)}{\min _{\substack{i=1, \cdots, l \\
j=1, \cdots, n_{n}}} r_{j i}^{2}} \\
& \forall i=1, \cdots, k \\
& C_{i}>2 A \frac{\max _{c \in \Gamma} \max _{j=1, \cdots, n}\left[E_{1}(c)-E_{1}(c(j))\right]}{\frac{1}{\alpha_{i}}\left[\ln \left(d_{i} o p_{i} 1\right)-\ln \left(d_{i}\right)\right]^{2}}
\end{aligned}
$$

where $\Gamma$ is the set of feasible solutions and $o p_{i}$ an addition if the constraint is of the type $w_{i}^{t} x \leq d_{i}$ or subtraction if the constraint is of the type $w_{i}^{t} x \geq d_{i}$, then any vertex of the hypercube violating at least one constraint and including in its neighborhood a feasible solution can not be a local minimum for Ecam.

Proof: Let $c \in \Gamma$ and $c(j) \notin \Gamma$ violating at least an equality constraint, say $p$, or an inequality constraint, say $q$. Obviously, the vertex $c(j)$ is not a local minimum if the weights $A, B$, and $C_{i}$ take values such that $\operatorname{Ecam}(c(j))>$
$\operatorname{Ecam}(c)$. That is

$$
\begin{aligned}
& A\left[E_{1}(c(j))-E_{1}(c)\right]+B\left[E_{2}(c(j))-E_{2}(c)\right] \\
& \quad+\left[\operatorname{Ecam}_{3}(c(j))-E_{c a m}(c)\right]>0 .
\end{aligned}
$$

On the vertex $c(j)$ violating the $p$ th equality constraint the term $E_{2}(c(j))$ is increased with regard to $E_{2}(c)$ by at least a cost $P_{e_{p}}$ equal to

$$
\begin{aligned}
P_{e_{p}}= & \frac{1}{2}\left[r_{p}^{t} c(j)\right]^{2}-s_{p} \times r_{p}^{t} c(j)-\frac{1}{2}\left[r_{p}^{t} c\right]^{2}+s_{p} \times r_{p}^{t} c \\
= & \left.r_{p}^{t} c+r_{j p}\left(c(j)_{j}-c_{j}\right)\right] \\
& \times\left[\frac{1}{2}\left(r_{p}^{t} c+r_{j p}\left[c(j)_{j}-c_{j}\right]\right)-s_{p}\right]+\frac{1}{2} s_{p}^{2} \\
& \quad\left(\text { since, } r_{p}^{t} c=s_{p}\right) \\
= & {\left[s_{p}+r_{j p}\left[1-2 c_{j}\right]\right] \times\left[\frac{1}{2}\left(s_{p}+r_{j p}\left[1-2 c_{j}\right]\right)-s_{p}\right] } \\
& +\frac{1}{2} s_{p}^{2} \quad\left(\text { since, }, c(j)_{j}=1-c_{j}\right) \\
= & {\left[s_{p}+r_{j p}\left[1-2 c_{j}\right]\right] \times\left[\frac{1}{2} r_{j p}\left[1-2 c_{j}\right]-\frac{1}{2} s_{p}\right]+\frac{1}{2} s_{p}^{2} } \\
= & \frac{1}{2} r_{j p}^{2} \quad\left(\text { since, },\left(1-2 c_{j}\right)^{2}=1\right) .
\end{aligned}
$$

So, we have $E_{2}(c(j))-E_{2}(c) \geq \frac{1}{2} r_{j p}^{2}$ and $\operatorname{Ecam}_{3}(c(j)) \geq$ $\operatorname{Ecam}_{3}(c)=0$. If the weight $B$ is such that $B>2 A\left[E_{1}(c)-\right.$ $\left.E_{1}(c(j))\right] / r_{j p}^{2}$, then (20) is always verified.

On $c(j)$ violating the $q$ th inequality constraint the energy $\operatorname{Ecam}_{3}(c(j))$ is at least equal to

$$
P_{i_{q}}=\frac{1}{2} \frac{C_{q}}{\alpha_{q}}\left[\ln \left(w_{q}^{t} c(j)^{\alpha_{q}}\right)-d_{q}^{\prime} y(j)_{q}\right]^{2} .
$$

A study of the (EHM)cam dynamics (15) gives that $d_{q}^{\prime} y(j)_{q}$ converges asymptotically to $\ln \left(d_{q}\right)$. So, when the network reaches convergence, $P_{i_{q}}$ can be approximated by: $P_{i_{q}} \approx \frac{1}{2}\left(C_{q} / \alpha_{q}\right)\left[\ln \left(w_{q}^{t} c(j)^{\alpha_{q}}\right)-\ln \left(d_{q}\right)\right]^{2}$. Since $w_{q}^{t} c(j)^{\alpha_{q}}$ is bounded below by $d_{q}+1$ if $w_{q}^{t} x \leq d_{q}$ and upper bounded by $d_{q}-1$ if $w_{q}^{t} x \geq d_{q}$ (we recall that $w_{j q}$ and $d_{q}$ are integers), we obtain that

$$
P_{i_{q}} \geq\left\{\begin{array}{l}
\frac{1}{2} \frac{C_{q}}{\alpha_{q}}\left[\ln \left(d_{q}+1\right)-\ln \left(d_{q}\right)\right]^{2}, \\
\text { if the type of the constraint is } w_{q}^{t} x \leq d_{q} \\
\frac{1}{2} \frac{C_{q}}{\alpha_{q}}\left[\ln \left(d_{q}-1\right)-\ln \left(d_{q}\right)\right]^{2}, \\
\text { if the type of the constraint is } w_{q}^{t} x \geq d_{q} .
\end{array}\right.
$$

Since $c \in \Gamma$, we have $\operatorname{Ecam}_{3}(c)=0$ and $E_{2}(c(j)) \geq E_{2}(c)$. So, by taking the weights $A$ and $C_{q}$ as indicated in (19), (20) is verified.

Notice that the weight $C_{q}$ must be chosen at least equal to the inverse of the minimal cost

$$
P_{i_{q}}=\frac{1}{2} \frac{1}{\alpha_{q}}\left[\ln \left(d_{q} o p_{q} 1\right)-\ln \left(d_{q}\right)\right]^{2}, \quad q=1, \cdots, k .
$$

So, its value depends on the bounds $d_{q}$ and the type of the constraints which fix the operators $o p_{q}$.

Let us see the case $d_{q}=1$. If the constraint is lower bounded, i.e., $w_{q}^{t} x \geq 1$, then the operator $o p_{q}$ is a subtraction. Since the cost $P_{i_{q}}$ tends to infinity, the weight $C_{q}$ can take any positive value. If the constraint is upper bounded, i.e., $w_{q}^{t} x \leq 1$, the operator $o p_{q}$ is an addition and the cost $P_{i_{q}}$ becomes equal to $P_{i_{q}}=\frac{1}{2} 1 / \alpha_{q}[\ln (2)]^{2}$. It is clearly 
possible to find a value for the weight $C_{q}$ that satisfies condition (19). For the case $d_{q}>1$, for satisfying the condition (19), the weight $C_{q}$ must take large values since whatever the constraint type the quantities $\ln \left(d_{q}+1\right)-\ln \left(d_{q}\right)$ or $\ln \left(d_{q}-1\right)-\ln \left(d_{q}\right)$ are small. Obviously, the network dynamic will be principally controlled by the constraints and less by the objective function. Consequently, fixing a value for the weight $C_{q}$ is possible only when the global amount $\sum_{j}$ out $_{\Upsilon_{i} \rightarrow \Xi_{j}}$ of flows emitting by the neuron $\Upsilon_{q}$ is sufficiently large as long as its associated constraint remains unsatisfied. The neperien logarithm function is well adapted when the (EHM)cam must handle inequality constraint of type $w_{i}^{t} x \geq 1$. For the constraints $w_{i}^{t} x \leq 1$, it would be necessary in practice to use a logarithm with a smaller base in order to reduce the weight $C_{q}$. On the other hand, for the inequality constraints with large bound $d_{i}$ the alone introduction of the logarithm function is rather unadvised. We can use it, for example, with a hyperbolic tangent. This function preserves around the bound $d_{i}$ a sufficient emitting of flows. Nevertheless, the choice of any other function different to the logarithm will imply the lost of the equality between the global quantity of flows emitting by a neuron and the amount of its resource. However, a such choice must preserve the two other principles of cam.

\section{The Set Covering Problem}

We consider a set $Q$ with $k$ elements and a collection $R$ of $n$ weighted subsets of $Q$. For $j=1, \cdots, n$ the $j$ th set is labeled $S_{j}$, and its weight $c_{j}$. Each element $q_{i}$ of $Q$ is supposed being included to at least one set of the collection $R$. The exact composition of each set is given by the $k \times n$ matrix $W: w_{j i}$ is equal to one if the $i$ th element belongs to the $j$ th set, and zero otherwise.

A cover is a subcollection $S$ of sets from $R$ not necessarily disjoint which covers all elements of $Q$, i.e., such that every element of $Q$ belongs to, at least, one member of $S$. The weighted minimum set covering problem (WSCP) consists in finding a cover minimizing the total weight $\sum_{S_{j} \in S} c_{j}$ for a given instance $I=(R, Q)$. This problem is known to be NPhard [7] and it can be formulated as an integer linear program as follows:

$$
\begin{aligned}
& \operatorname{minimize} c^{t} x \\
& \text { subject to: } w_{i}^{t} x \geq 1 \quad \forall i=1, \cdots, k \\
& \text { with } x \in\{0,1\}^{n} .
\end{aligned}
$$

In order to evaluate the quality of neural solutions we use the greedy algorithm due to Johnson [6] as a comparison measure. This algorithm chooses at each step the set $S_{j}$ with the larger ratio $\left|S_{j}\right| / c_{j}$ to put in the solution as long as all elements of $Q$ are not covered. Next, the introduced set and their elements are removed from $R$ and $Q$ and the cardinalities of the other sets are updated. With such an algorithm solutions minimality and sets irredundance are not always guaranteed. A worst case tight bound has been established by Johnson [6] and Chvátal [3] equal to $\sum_{j=1}^{d}(1 / j)$ times the weight of an optimal cover, where $d$ is the size of the largest set $S_{j}$. This approximation ratio is reachable for a series of particular unweighted instances. We use a such instance for evaluating the performance of EHM and (EHM)cam neural networks.

In the EHM, the covering constraints are introduced by the penalty function

$$
E_{3}=\frac{1}{2} \sum_{i=1}^{k}\left[w_{i}^{t} x-y_{i}\right]^{2} \quad \text { with } y_{i} \geq 1, i=1, \cdots, k .
$$

The derived activation rules [(7) and (8)] are

$\diamond$ for the sets $S_{j}$

$$
d x_{j} / d t=\left(C \sum_{i=1}^{k} w_{j i} x_{j}\left(y_{i}-w_{i}^{t} x\right)-A c_{j} x_{j}\right)\left(1-x_{j}\right)
$$

$\diamond$ for the elements $q_{i}: d y_{i} / d t=-C\left(y_{i}-1\right)\left(y_{i}-w_{i}^{t} x\right)$, with $C>A \max _{j=1}, \cdots, n c_{j}$.

In the (EHM)cam, the covering constraints are introduced by the function

$$
\operatorname{Ecam}_{3}=\frac{1}{2} \sum_{i=1}^{k} \frac{C_{i}}{\alpha_{i}}\left[\ln \left(w_{i}^{t} x^{\alpha}\right)-y_{i}\right]^{2}
$$

with $\alpha_{i}>1$ and $y_{i} \geq 0, i=1, \cdots, k$.

As the $k$ constraints are the same, we take $C_{i}=C$ and $\alpha_{i}=\alpha \forall i=1, \cdots, k$ (with $C>0$ and $\alpha>1$ ). The derived activation rules [(14) and (15)] are

$\diamond$ for the sets $S_{j}$

$$
\begin{aligned}
d x_{j} / d t= & {\left[C \sum_{i=1}^{k} \frac{w_{j i} x_{j}^{\alpha}}{w_{i}^{t} x^{\alpha}}\left(y_{i}-\ln \left(w_{i}^{t} x^{\alpha}\right)\right)-A c_{j} x_{j}\right] } \\
& \times\left(1-x_{j}\right)
\end{aligned}
$$

$\diamond$ for the elements $q_{i}$

$$
d y_{i} / d t=-C / \alpha y_{i}\left(y_{i}-\ln \left(w_{i}^{t} x^{\alpha}\right)\right) .
$$

By (19), the weight $C$ can take any positive value and $A$ can be fixed to one.

\section{EXPERIMENTAL RESULTS}

In order to extract the cover from the state of the network at convergence we consider two experimentally established thresholds $L^{\text {inf }}$ and $L^{\text {sup }}$ fixed, respectively, to the values 0.3 and 0.7. The first one is the threshold below which the neuron is considered as inactive. The second is the threshold up which the neuron is active. Any activation level located between these two thresholds is considered as intermediate and so, it is ambiguous to interpret.

To break the symmetry and thus prevent the system from settling down into an unstable equilibrium state, the initial activation levels are randomly attributed in a fixed interval. A weak width for this interval gives equivalent chances for any set to belong to the cover. The interval chosen has a width equal to 0.1 centered around 0.5 . In our experiments we have considered that the model reaches convergence when two consecutive states are almost identical. Their differences are measured by the evolution rate [9]: $\Lambda=(1 / n) \sum_{j=1}^{n}\left[x_{j}(t+\right.$ $\left.\Delta t)-x_{j}(t)\right]^{2}$. We have fixed the limit for $\Lambda$ equal to $10^{-10}$. The time step $\Delta t$ was equal to $\Delta t=0.01$. This large time 
step has sometimes as effect to violate the activation bounds. In such cases, the activations are reset nearer the bounds zero or one. The weights were fixed to $A=1$ and $C=2$ which also are valid for the EHM (see Section IV).

The first objective of our experiments was to compare the two versions of the extended Hopfield model on their capacity to share out the neurons corresponding to the sets of the collection $R$. We have considered a randomly generated instance containing 60 sets and 30 elements. The Fig. 1 reports, for the EHM and for the (EHM)cam, the evolution of the activation levels $x_{j}, j=1, \cdots, n$. For the (EHM)cam different values of the power $\alpha$ were studied.

For the EHM, case $a$, we observe the existence of a large number of neurons with ambiguous interpretation. So, a cover cannot be deduced from the final state. For the (EHM)cam, cases $b, c$, and $d$, the repartition of the sets into two active/inactive categories is realized as soon as $\alpha>1$. For $\alpha=2$ (case $c$ ), about a thousand iterations are needed. For $\alpha=3$ (case $d$ ), a hundred of iterations are sufficient to yield a cover. But, the rapidity of convergence is achieved in prejudice of the solution quality. Indeed, the cardinality of the solution returned by the model for $\alpha=3$ is more expensive than the one obtained for $\alpha=2$ (11 sets instead of nine). It is clear that when the convergence is too fast, the favor conceded by an element is so great for the most active neurons that any weak difference generated during the initialization phase affects the solution. Our study with different values of $\alpha$ has shown that the best experimental behavior is achieved for the (EHM)cam when the parameter $\alpha=2$. The sets are clearly shared out and the final solution is relatively not conditioned by the initialization interval.

The second part of our experimental study consists in investigating the behavior of EHM and (EHM) cam for $\alpha=2$ on some particular instances of Johnson. We have observed a clear repartition of the neurons into active and inactive by both models. However, the (EHM) cam converges faster than EHM. The final state for both models is composed exclusively by neurons giving the optimal solution. We see that such instances, which are difficult for the sequential heuristic, become easy for the studied neural networks.

In the third part, we evaluate the (EHM) cam on its capacity of settling down into states without intermediate activations and its solution quality in comparison with the greedy algorithm. We do not report results on the EHM since, for many treated instances, many neurons had involved with ambiguous classification. By considering the same hard limits $L^{\mathrm{inf}}(0.3)$ and $L^{\mathrm{sup}}(0.7)$ the produced solutions were not enough interesting.

We have considered different groups of 50 randomly generated unweighted graphs. They are specified by giving (see Table I).

- The size of the instance $I=(R, Q)$ defined by the number $|R|$ of sets composing the collection $R$ and the number $|Q|$ of elements included in the basic set $Q$.

- The number $\left|q_{i}\right|$ of sets containing an element of $Q$. An interval of authorized values is given for this parameter.

- The maximum number of elements included into a set $S_{j}$ of the collection $R$. In order to make the competitions
EHM

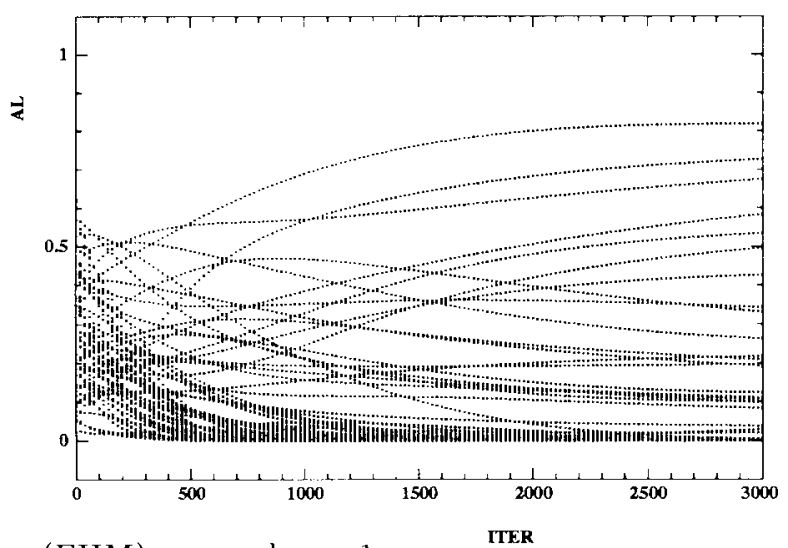

(EHM)cam and $\alpha=1$

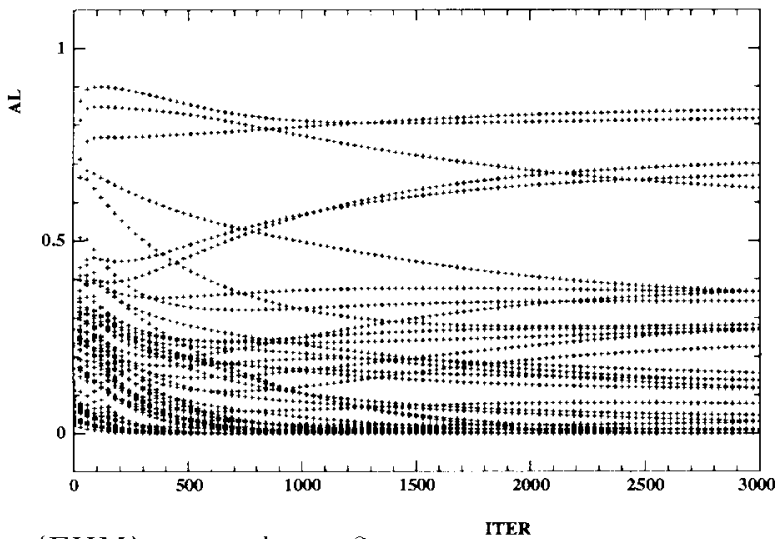

(EHM)cam and $\alpha=2$

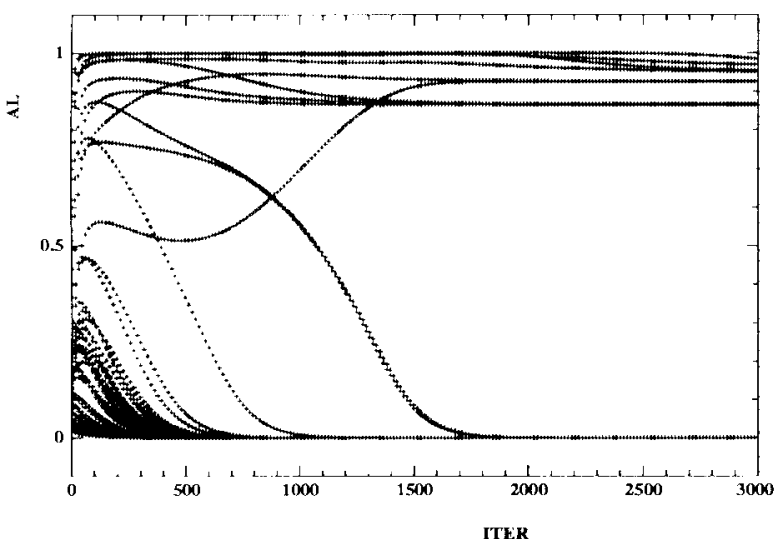

(EHM)cam and $\alpha=3$

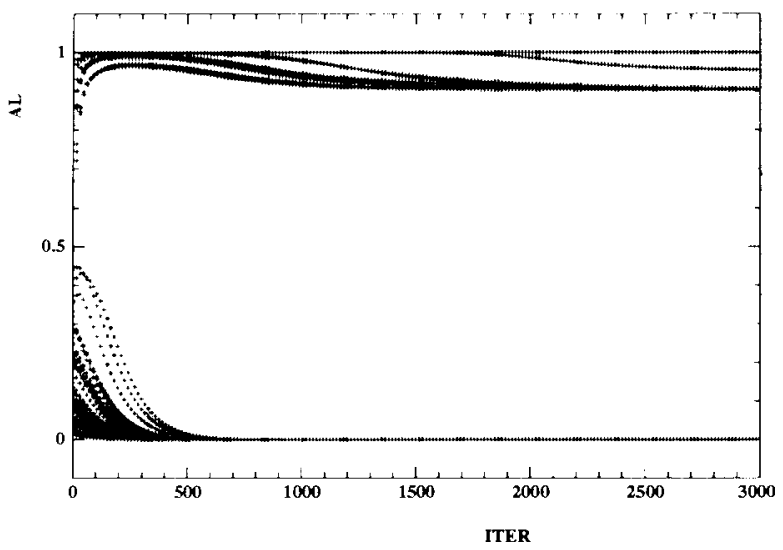

Fig. 1. Activation level (AL) evolution in function of the number of iterations (ITER) for the sets of a randomly generated instance. 
TABLE I

Neural Network (NN) Performance (Solution Quality ANd Average CPU Time in Seconds) in Comparison to A Heuristic $(\mathrm{H})$ for Different Instance Sizes ANd Densities

\begin{tabular}{|c|c|c|c|c|c|c|}
\hline$\left|q_{i}\right|$ & $|R| \times|Q|$ & $\mathrm{H}$ & $=$ & NN & $T_{H}$ & $T_{N N}$ \\
\hline \multirow{5}{*}[1..5]{} & 8040 & 6 & 28 & 66 & 0.1 & 15.3 \\
\hline & 10030 & 6 & 42 & 52 & 0.09 & 12.5 \\
\hline & 10050 & 0 & 36 & 64 & 0.17 & 25.5 \\
\hline & 15050 & 6 & 24 & 70 & 0.37 & 34.8 \\
\hline & average & 4.5 & 32.5 & 63.0 & & \\
\hline \multirow{5}{*}[3..5]{} & 8040 & 4 & 28 & 68 & 0.09 & 21.3 \\
\hline & 10030 & 4 & 26 & 70 & 0.09 & 15 \\
\hline & 10050 & 14 & 38 & 48 & 0.16 & 32.4 \\
\hline & 15050 & 2 & 18 & 80 & 0.25 & 41.7 \\
\hline & average & 6.0 & 27.5 & 66.5 & & \\
\hline \multirow{5}{*}[3..7]{} & 8040 & 12 & 34 & 54 & 0.09 & 25.1 \\
\hline & 10030 & 12 & 34 & 54 & 0.08 & 16.4 \\
\hline & 10050 & 16 & 34 & 50 & 0.15 & 37.5 \\
\hline & 15050 & 4 & 12 & 84 & 0.25 & 44.3 \\
\hline & average & 11.0 & 28.5 & 60.5 & & \\
\hline \multirow{5}{*}[3..10]{} & 8040 & 18 & 34 & 48 & 0.09 & 23.3 \\
\hline & 10030 & 12 & 36 & 52 & 0.08 & 17.3 \\
\hline & 10050 & 12 & 18 & 70 & 0.14 & 42.3 \\
\hline & 15050 & 16 & 22 & 62 & 0.22 & 57.1 \\
\hline & average & 14.5 & 27.5 & 58.0 & & \\
\hline \multirow{5}{*}[5..10]{} & 8040 & 24 & 38 & 38 & 0.08 & 29.9 \\
\hline & 10030 & 14 & 42 & 44 & 0.08 & 18.6 \\
\hline & 10050 & 22 & 24 & 54 & 0.14 & 50.8 \\
\hline & 15050 & 12 & 24 & 64 & 0.22 & 60.8 \\
\hline & average & 18.0 & 32.0 & 50.0 & & \\
\hline
\end{tabular}

more uncertain this number was limited to seven for each instance.

Table I gives the performance of the two methods from solution quality point of view and CPU required time. For each group of instances, the percentages of the cases where the heuristic has given a better solution than the neural network (column "H"), an equivalent solution (column “=”), and finally a worst solution (column "NN") are reported. Also the average required CPU times (on a SPARC 10) for the heuristic $T_{H}$ and the neural network $T_{N N}$ are presented.

Even with the instituted cam in some cases the network settles into a state with some neurons having intermediate activations. The percentage of such solutions increases with the average degree of the basic elements, but at average never it exceeds $20 \%$. More often the number of concerned neurons is about three or five but never exceeds the eight. These neurons with intermediate activations are considered as active and are introduced into the solution. This has always given feasible solutions but had introduced some redundant sets.

For a percentage of cases ranging between $50-66.5 \%$, the $\mathrm{NN}$ presents a strictly better performance than $\mathrm{H}$. The most favorable cases correspond to the groups of instances for which few number of sets contain each element of $Q$, i.e., $\left|q_{i}\right| \in[1 \cdots 5]$ or $\left|q_{i}\right| \in[3 \cdots 5]$. Two reasons explain the excellent results of the $\mathrm{NN}$ for those groups of instances. Firstly, because very few solutions (about $8 \%$ instead of 14-20\% for other groups) contain neurons with intermediate activations. The second reason is the low efficiency of $\mathrm{H}$ when the instance is made up of some basic elements included only in one set. Obviously, the insertion of these sets into the cover is necessary and if they have a weak cardinality the greedy algorithm introduces them only during the last steps. It can be seen, in Table I, that for high densities, i.e., $\left|q_{i}\right| \in$ $[3 \cdots 7],\left|q_{i}\right| \in[3 \cdots 10]$, or $\left|q_{i}\right| \in[5 \cdots 10]$, the NN gives a strictly better solution than $\mathrm{H}$ in a percentage of cases ranging between $50-60 \%$, and an equivalent solution in about $30 \%$ of cases. A degradation of the NN performance is observed when the density increases. However, the NN performs still better than $\mathrm{H}$. In the worst case $\left(\left|q_{i}\right| \in[5 \cdots 10]\right)$, a better cover is given by the $\mathrm{NN}$ in $50 \%$ of cases, comparatively to only $18 \%$ for the $\mathrm{H}$.

Notice that the NN requires larger CPU time than $\mathrm{H}$ but it remains at average lower than one minute. This time would sometimes be acceptable if solutions quality is the main objective while a massively parallel implementation let us expecting significant improvements.

\section{CONCLUSION}

We have proposed a new expression for the penalty energy handling inequality constraints in Hopfield models. The derived rules introduce competitions between the variables involved into the same constraint and solve them with the competitive activation mechanism. The treatment of the upper bounded inequality constraints generates competitions for which the objective is negative resources acquisition. In consequence, we have extended the competitive activation mechanism for dealing also with negative resources. The great interest for this mechanism is its capacity to distribute the neurons into two active/inactive categories. This point remedies a drawback of the extended Hopfield models. Validation was given through an extensive experimental study on the set cover problem.

\section{ACKNOWLEDGMENT}

The authors would like to thank an anonymous referee for several valuable suggestions.

\section{REFERENCES}

[1] S. Abe, "Theories on the Hopfield neural networks," in Proc. Int. Joint Conf. Neural Networks, Washington, D.C., 1989, vol. I, pp. 557-564.

[2] S. Abe, J. Kawakami, and K. Hirasawa, "Solving inequality constrained combinatorial optimization problems by the Hopfield neural networks," Neural Networks, vol. 5, pp. 663-670, 1992.

[3] V. Chvátal, "A greedy heuristic for the set-covering problem," Math Operations Res., vol. 4, no. 3, pp. 233-235, 1979.

[4] S. Grossberg, Studies of Mind and Brain: Neural Principles of Learning, Perception, Development, Cognition, and Motor Control. Boston, MA: Reidel, 1982.

[5] J. J. Hopfield and D. W. Tank, "Neural computation of decisions in optimization problems," Biol. Cybern., vol. 52, pp. 141-152, 1985.

[6] D. S. Johnson, "Approximation algorithms for combinatorial problems," J. Comput. Syst. Sci., vol. 9, pp. 256-278, 1974.

[7] R. M. Karp, "Reducibility among combinatorial problems," Complexity of Computer Computations. New York: Plenum, 1972, pp. 85-104.

[8] A. Le Gall and V. Zissimopoulos, "A competitive activation neuralnetwork model for the weighted minimum vertex covering, "Int. J. Neural Syst., vol. 7, pp. 607-616, 1996.

[9] M. Ohlsson, C. Peterson, and B. Soderberg, "Neural networks for optimization problems with inequality constraints-The knapsack problem," Neural Comput., vol. 5, pp. 331-339, 1993. 
[10] M. Ohlsson and H. Pi, "A study of the mean field approach to knapsack problems," Neural Networks, vol. 10, pp. 263-271, 1997.

[11] C. Peterson and B. Söderberg, "A new method for mapping optimization problems onto neural networks," Int. J. Neural Syst., vol. 1, no. 1, pp. $3-22,1989$

[12] J. A. Reggia, "Properties of a competition-based activation mechanism in neuromimetic networks models," in Proc. 1st Int. Conf. Neural Networks, 1987, vol. II, pp. 131-138.

[13] J. A. Reggia, Y. Peng, and P. Bourret, "Recent applications of competitive activation mechanisms," Neural Networks: Advances and Applications, pp. 33-62, 1991.

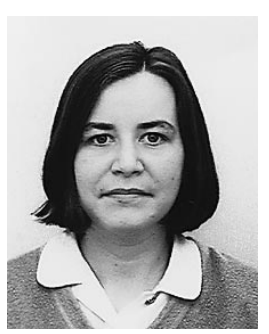

Armelle Le Gall received the Ph.D. degree in computer science from the University of Paris-Sud in 1997.

She is a Research Assistant at the University of Paris-Nord. Her research interests are incremental and adaptive algorithms for solving combinatorial optimization problems and neural networks.

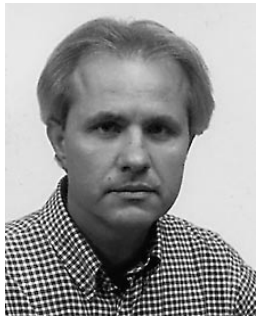

Vassilis Zissimopoulos received the bachelor's degree from Athens University, Greece, and the Ph.D. degree in computer science at the University of Paris-Sud in 1984

$\mathrm{He}$ is currently Professor of computer science at the University of Paris-Nord. His research interests include approximation algorithms, local search, neural networks in combinatorial optimization, incremental algorithms, task allocation, static and dynamic scheduling, load balancing, and adaptive algorithms. 